\title{
An Assessment of Theoretical Procedures for Predicting the Thermochemistry and Kinetics of Hydrogen Abstraction by Methyl Radical from Benzene
}

\author{
Karen Hemelsoet, ${ }^{\dagger, \star}$ Damian Moran,,$*$ Veronique Van Speybroeck, ${ }^{*} \dagger$ \\ Michel Waroquier ${ }^{\dagger}$ and Leo Radom ${ }^{*}$, , \\ Center for Molecular Modeling, Ghent University, Proeftuinstraat 86, B-9000 Gent, Belgium, \\ School of Chemistry, University of Sydney, NSW 2006, Australia and ARC Centre of Excellence in \\ Free Radical Chemistry and Biotechnology
}

\section{SUPPORTING INFORMATION}

(Table S1-S7, Total 18 pages)

\footnotetext{
*To whom correspondence should be addressed. E-mail: dmoran@chem.usyd.edu.au; veronique.vanspeybroeck@ugent.be; radom@chem.usyd.edu.au ${ }^{\dagger}$ Ghent University *University of Sydney

"ARC Centre of Excellence in Free Radical Chemistry and Biotechnology
} 
TABLE S1: Optimized Geometries (Cartesian Coordinates, Å) for Benzene, Phenyl Radical, Methyl Radical, and Methane at the B3-LYP, BMK and CCSD Levels with the 6$31+G(d, p)$ Basis Set

\begin{tabular}{|c|c|c|c|c|c|c|c|c|}
\hline & \multicolumn{4}{|c|}{$\mathrm{C}_{6} \mathrm{H}_{6}$} & \multicolumn{4}{|c|}{$\mathrm{C}_{6} \mathrm{H}_{5^{\bullet}}$} \\
\hline \multirow[t]{12}{*}{ B3-LYP } & $\mathrm{C}$ & -0.012491 & 0.000000 & -0.007210 & $\mathrm{C}$ & -0.011384 & 0.000000 & -0.009973 \\
\hline & $\mathrm{C}$ & -0.012491 & 0.000000 & 1.391129 & $\mathrm{C}$ & -0.012370 & 0.000000 & 1.396329 \\
\hline & $\mathrm{C}$ & 1.198506 & 0.000000 & 2.090299 & $\mathrm{C}$ & 1.196565 & 0.000000 & 2.100751 \\
\hline & $\mathrm{C}$ & 2.409504 & 0.000000 & 1.391129 & $\mathrm{C}$ & 2.418729 & 0.000000 & 1.419544 \\
\hline & $\mathrm{C}$ & 2.409504 & 0.000000 & -0.007210 & $\mathrm{C}$ & 2.444600 & 0.000000 & 0.013479 \\
\hline & $\mathrm{C}$ & 1.198506 & 0.000000 & -0.706380 & $\mathrm{C}$ & 1.222593 & 0.000000 & -0.625013 \\
\hline & $\mathrm{H}$ & 1.198506 & 0.000000 & 3.176708 & $\mathrm{H}$ & -0.941639 & 0.000000 & -0.570649 \\
\hline & $\mathrm{H}$ & 3.350362 & 0.000000 & 1.934334 & $\mathrm{H}$ & -0.957592 & 0.000000 & 1.933242 \\
\hline & $\mathrm{H}$ & 3.350362 & 0.000000 & -0.550415 & $\mathrm{H}$ & 1.186195 & 0.000000 & 3.186696 \\
\hline & $\mathrm{H}$ & 1.198506 & 0.000000 & -0.792789 & $\mathrm{H}$ & 3.353527 & 0.000000 & 1.974408 \\
\hline & $\mathrm{H}$ & -0.953349 & 0.000000 & -0.550415 & $\mathrm{H}$ & 3.385393 & 0.000000 & -0.529329 \\
\hline & $\mathrm{H}$ & -0.953349 & 0.000000 & 1.934334 & & & & \\
\hline \multirow[t]{12}{*}{ BMK } & $\mathrm{C}$ & 0.000000 & -1.401914 & 0.000000 & $\mathrm{C}$ & 0.000000 & 0.000000 & 1.329190 \\
\hline & $\mathrm{C}$ & -1.214093 & -0.700957 & 0.000000 & $\mathrm{C}$ & 0.000000 & 1.218469 & 0.634075 \\
\hline & $\mathrm{C}$ & -1.214093 & 0.700957 & 0.000000 & $\mathrm{C}$ & 0.000000 & 1.231568 & -0.775674 \\
\hline & $\mathrm{C}$ & 0.000000 & 1.401914 & 0.000000 & $\mathrm{C}$ & 0.000000 & 0.000000 & -1.405084 \\
\hline & $\mathrm{C}$ & 1.214093 & 0.700957 & 0.000000 & $\mathrm{C}$ & 0.000000 & -1.231568 & -0.775674 \\
\hline & $\mathrm{C}$ & 1.214093 & -0.700957 & 0.000000 & $\mathrm{C}$ & 0.000000 & -1.218469 & 0.634075 \\
\hline & $\mathrm{H}$ & 0.000000 & -2.490395 & 0.000000 & $\mathrm{H}$ & 0.000000 & -2.167891 & -1.329593 \\
\hline & $\mathrm{H}$ & -2.156745 & -1.245197 & 0.000000 & $\mathrm{H}$ & 0.000000 & -2.160143 & 1.181102 \\
\hline & $\mathrm{H}$ & -2.156745 & 1.245197 & 0.000000 & $\mathrm{H}$ & 0.000000 & 0.000000 & 2.417208 \\
\hline & $\mathrm{H}$ & 0.000000 & 2.490395 & 0.000000 & $\mathrm{H}$ & 0.000000 & 2.160143 & 1.181102 \\
\hline & $\mathrm{H}$ & 2.156745 & 1.245197 & 0.000000 & $\mathrm{H}$ & 0.000000 & 2.167891 & -1.329593 \\
\hline & $\mathrm{H}$ & 2.156745 & -1.245197 & 0.000000 & & & & \\
\hline
\end{tabular}


TABLE S1: continued ...

\begin{tabular}{|c|c|c|c|c|c|c|c|c|}
\hline \multirow{13}{*}{ CCSD } & $\mathrm{C}$ & -0.012940 & 0.000000 & -0.007469 & $\mathrm{C}$ & -0.010571 & 0.000000 & -0.006791 \\
\hline & $\mathrm{C}$ & -0.012940 & 0.000000 & 1.391388 & $\mathrm{C}$ & -0.010351 & 0.000000 & 1.395106 \\
\hline & $\mathrm{C}$ & 1.198506 & 0.000000 & 2.090817 & $\mathrm{C}$ & 1.196581 & 0.000000 & 2.098984 \\
\hline & $\mathrm{C}$ & 2.409953 & 0.000000 & 1.391388 & $\mathrm{C}$ & 2.416735 & 0.000000 & 1.418283 \\
\hline & $\mathrm{C}$ & 2.409953 & 0.000000 & -0.007469 & $\mathrm{C}$ & 2.443727 & 0.000000 & 0.016644 \\
\hline & $\mathrm{C}$ & 1.198506 & 0.000000 & -0.706898 & $\mathrm{C}$ & 1.222620 & 0.000000 & -0.627798 \\
\hline & $\mathrm{H}$ & 1.198506 & 0.000000 & 3.173781 & $\mathrm{H}$ & -0.936912 & 0.000000 & -0.565975 \\
\hline & $\mathrm{H}$ & 3.347827 & 0.000000 & 1.932870 & $\mathrm{H}$ & -0.951562 & 0.000000 & 1.931614 \\
\hline & $\mathrm{H}$ & 3.347827 & 0.000000 & -0.548951 & $\mathrm{H}$ & 1.186244 & 0.000000 & 3.181501 \\
\hline & $\mathrm{H}$ & 1.198506 & 0.000000 & -1.789862 & $\mathrm{H}$ & 3.347528 & 0.000000 & 1.972666 \\
\hline & $\mathrm{H}$ & -0.950815 & 0.000000 & -0.548951 & $\mathrm{H}$ & 3.380577 & 0.000000 & -0.524747 \\
\hline & $\mathrm{H}$ & -0 & 0.000000 & 70 & & & & \\
\hline & \multicolumn{4}{|c|}{$\mathrm{CH}_{3}{ }^{\bullet}$} & \multicolumn{4}{|c|}{$\mathrm{CH}_{4}$} \\
\hline \multirow[t]{5}{*}{ B3-LYP } & C & -0.000058 & 0.000024 & -0.000062 & $\mathrm{C}$ & 0.000000 & 0.000000 & 0.000000 \\
\hline & $U$ & 0.000064 & -0.000009 & 1.082669 & $\mathrm{H}$ & 0.000000 & 0.000000 & 1.092795 \\
\hline & $\mathrm{H}$ & 0.937487 & -0.000009 & -0.541653 & $\mathrm{H}$ & 1.030297 & 0.000001 & -0.364265 \\
\hline & $\mathrm{H}$ & -0.937826 & 0.001791 & -0.541289 & $\mathrm{H}$ & -0.515148 & -0.892263 & -0.364265 \\
\hline & & & & & $\mathrm{H}$ & -0.515149 & 0.892263 & -0.364265 \\
\hline \multirow[t]{5}{*}{ BMK } & $c$ & 0.00 & 0.00 & 0.000000 & $\mathrm{C}$ & 0.000000 & 0.000000 & 0.000000 \\
\hline & $\mathrm{H}$ & 0.000000 & 1.085644 & 0.000000 & $\mathrm{H}$ & 0.000000 & 0.000000 & 1.094969 \\
\hline & $\mathrm{H}$ & 0.940195 & -0.542822 & 0.000000 & $\mathrm{H}$ & 0.000000 & -1.032346 & -0.364990 \\
\hline & $\mathrm{H}$ & -0.940195 & -0.542822 & 0.000000 & $\mathrm{H}$ & -0.894038 & 0.516173 & -0.364990 \\
\hline & & & & & $\mathrm{H}$ & 0.894038 & 0.516173 & -0.364990 \\
\hline \multirow[t]{5}{*}{ CCSD } & $\mathrm{C}$ & -0.0 & 0.000022 & -0.000061 & $\mathrm{C}$ & 0.000000 & 0.000000 & 0.000000 \\
\hline & $\mathrm{H}$ & 0.000069 & -0.000006 & 1.077866 & $\mathrm{H}$ & 0.000000 & 0.000000 & 1.088155 \\
\hline & $\mathrm{H}$ & 0.933329 & -0.000006 & -0.539247 & $\mathrm{H}$ & 1.025922 & 0.000001 & -0.362718 \\
\hline & $\mathrm{H}$ & -0.933669 & 0.001787 & -0.538892 & $\mathrm{H}$ & -0.512961 & -0.888475 & -0.362719 \\
\hline & & & & & $\mathrm{H}$ & -0.512961 & 0.888475 & -0.362718 \\
\hline
\end{tabular}


TABLE S2: Effect of DFT Integration Grid Density on Enthalpies and Barriers (kJ mol ${ }^{-1}$ ) and Pre-Exponential Factors $\left(\mathrm{m}^{3} \mathrm{~mol}^{-1} \mathrm{~s}^{-1}\right)$

\begin{tabular}{cccccc}
\hline & Grid Density & $\boldsymbol{\Delta H}$ & $\boldsymbol{\Delta} \mathbf{E}_{\mathbf{0}}^{\ddagger}$ & $\mathbf{E}_{\mathbf{a}}(\mathbf{F R} / \mathbf{H O})$ & $\mathbf{A}(\mathbf{H O} / \mathbf{F R})$ \\
\hline \multirow{2}{*}{ B3-LYP } & Standard (75,302)p & 19.1 & 62.3 & 67.1 & $3.03 \times 10^{7}$ \\
& UltraFine (99,590)p & 19.1 & 62.2 & 67.2 & $3.07 \times 10^{7}$ \\
\multirow{2}{*}{ BP86 } & Standard (75,302)p & 13.0 & 42.3 & 47.2 & $3.75 \times 10^{7}$ \\
& UltraFine (99,590)p & 13.0 & 42.3 & 47.1 & $3.77 \times 10^{7}$ \\
\hline
\end{tabular}


TABLE S3: Absolute Vibrationless Energies ( $E_{0}$, hartrees), Unscaled Zero-point Vibrational Energies (ZPVE, hartrees) and Thermal Corrections to the Enthalpy $(\Delta \Delta H$, hartrees) ${ }^{a}$

\begin{tabular}{|c|c|c|c|c|c|c|}
\hline & \multicolumn{3}{|c|}{$\mathrm{C}_{6} \mathrm{H}_{6}$} & \multicolumn{3}{|c|}{$\mathrm{CH}_{3}{ }^{\bullet}$} \\
\hline & $\mathbf{E}_{\mathbf{0}}$ & ZPVE & $\Delta \Delta \mathrm{H}$ & $\mathbf{E}_{\mathbf{0}}$ & ZPVE & $\Delta \Delta \mathrm{H}$ \\
\hline BP86/6-31G(d) & -232.23823 & 0.09778 & 0.10299 & -39.83120 & 0.02914 & 0.03312 \\
\hline $\mathrm{BP} 86 / 6-31+\mathrm{G}(\mathrm{d}, \mathrm{p})$ & -232.25597 & 0.09750 & 0.10325 & -39.83967 & 0.02913 & 0.03318 \\
\hline BLYP/6-31G(d) & -232.13236 & 0.09776 & 0.10322 & -39.80538 & 0.02913 & 0.03318 \\
\hline $\mathrm{BLYP} / 6-31+\mathrm{G}(\mathrm{d}, \mathrm{p})$ & -232.15390 & 0.09744 & 0.10292 & -39.81546 & 0.02913 & 0.03313 \\
\hline B3-P86/6-31G(d) & -233.00434 & 0.10111 & 0.10645 & -39.99947 & 0.02995 & 0.03399 \\
\hline B3-P86/6-31+G(d,p) & -233.02088 & 0.10081 & 0.10616 & -40.00720 & 0.02993 & 0.03392 \\
\hline B3-LYP/6-31G(d) & -232.24866 & 0.10075 & 0.10608 & -39.83829 & 0.02983 & 0.03388 \\
\hline B3-LYP/6-31G(d,p) & -232.25821 & 0.10061 & 0.10595 & -39.84288 & 0.02976 & 0.03380 \\
\hline B3-LYP/6-31+G(d,p) & -232.26840 & 0.10041 & 0.10576 & -39.84733 & 0.02982 & 0.03381 \\
\hline B3-LYP/6-311G(d,p) & -232.30855 & 0.10016 & 0.10550 & -39.85376 & 0.02957 & 0.03358 \\
\hline B3-PW91/6-31G(d) & -232.15904 & 0.10098 & 0.10632 & -39.82315 & 0.02990 & 0.03394 \\
\hline B3-PW91/6-31+G(d,p) & -232.17559 & 0.10068 & 0.10604 & -39.83097 & 0.02988 & 0.03388 \\
\hline MPW1K/6-31G(d) & -232.18406 & 0.10387 & 0.10909 & -39.82821 & 0.03060 & 0.03465 \\
\hline MPW1K/6-31+G(d,p) & -232.20020 & 0.10354 & 0.10878 & -39.83568 & 0.03056 & 0.03456 \\
\hline $\mathrm{BB} 1 \mathrm{~K} / 6-31 \mathrm{G}(\mathrm{d})$ & -232.12501 & 0.10387 & 0.10910 & -39.80300 & 0.03039 & 0.03447 \\
\hline $\mathrm{BB} 1 \mathrm{~K} / 6-31+\mathrm{G}(\mathrm{d}, \mathrm{p})$ & -232.14110 & 0.10352 & 0.10877 & -39.81095 & 0.03040 & 0.03441 \\
\hline MPWB1K/6-31G(d) & -232.12105 & 0.10360 & 0.10885 & -39.80091 & 0.03046 & 0.03454 \\
\hline MPWB1K/6-31+G(d,p) & -232.13773 & 0.10326 & 0.10852 & -39.80909 & 0.03047 & 0.03448 \\
\hline $\mathrm{BMK} / 6-31 \mathrm{G}(\mathrm{d})$ & -232.07618 & 0.10138 & 0.10668 & -39.79725 & 0.03006 & 0.03410 \\
\hline $\mathrm{BMK} / 6-31+\mathrm{G}(\mathrm{d}, \mathrm{p})$ & -232.09128 & 0.10090 & 0.10622 & -39.80428 & 0.03000 & 0.03398 \\
\hline $\mathrm{UHF} / 6-31 \mathrm{G}(\mathrm{d})$ & -230.70314 & 0.10766 & 0.11271 & -39.55899 & 0.03097 & 0.03517 \\
\hline $\mathrm{UHF} / 6-31+\mathrm{G}(\mathrm{d}, \mathrm{p})$ & -230.72183 & 0.10720 & 0.11226 & -39.56670 & 0.03099 & 0.03507 \\
\hline RHF/6-31G(d) & -230.70314 & 0.10766 & 0.11271 & -39.55477 & 0.03123 & 0.03536 \\
\hline $\mathrm{RHF} / 6-31+\mathrm{G}(\mathrm{d}, \mathrm{p})$ & -230.72183 & 0.10720 & 0.11226 & -39.56276 & 0.03071 & 0.03502 \\
\hline MP2/6-31G(d) & -231.45773 & 0.10063 & 0.10616 & -39.66875 & 0.03053 & 0.03463 \\
\hline $\mathrm{MP} 2 / 6-31+\mathrm{G}(\mathrm{d}, \mathrm{p})$ & -231.51917 & 0.09989 & 0.10580 & -39.69633 & 0.03080 & 0.03484 \\
\hline QCISD/6-31G(d) & -231.49657 & 0.10043 & 0.10587 & -39.68912 & 0.02999 & 0.03406 \\
\hline QCISD/6-31+G(d,p) & -231.55693 & 0.10050 & 0.10610 & -39.71728 & 0.03042 & 0.03444 \\
\hline $\mathrm{CCSD} / 6-31 \mathrm{G}(\mathrm{d})$ & -231.49490 & 0.10050 & 0.10600 & -39.68900 & 0.03000 & 0.03410 \\
\hline $\mathrm{CCSD} / 6-31+\mathrm{G}(\mathrm{d}, \mathrm{p})$ & -231.55556 & 0.10057 & 0.10618 & -39.71717 & 0.03044 & 0.03446 \\
\hline $\mathrm{CCSD} / 6-311 \mathrm{G}(\mathrm{d}, \mathrm{p})$ & -231.61276 & 0.09965 & 0.10518 & -39.72905 & 0.02975 & 0.03382 \\
\hline
\end{tabular}


TABLE S3: continued ...

\begin{tabular}{|c|c|c|c|c|c|c|}
\hline $\begin{array}{l}\text { UMP2/ } \\
6-311+G(3 d f, 2 p)\end{array}$ & -231.72042 & $-{ }^{b}$ & $-{ }^{b}$ & -39.73126 & $-{ }^{b}$ & $-{ }^{b}$ \\
\hline $\begin{array}{l}\text { RMP } 2 / \\
6-311+G(3 d f, 2 p)\end{array}$ & -231.72042 & $-{ }^{b}$ & $-b$ & -39.73168 & $-b$ & $-{ }^{b}$ \\
\hline $\begin{array}{l}\text { URCCSD/ } \\
6-311+G(3 d f, 2 p)\end{array}$ & -231.80786 & $-{ }^{b}$ & $-b$ & -39.75881 & $-b$ & $-{ }_{-b}$ \\
\hline G3-RAD & -232.17914 & - & - & -39.82737 & - & - \\
\hline G3(MP2)-RAD & -231.98376 & - & - & -39.79460 & - & - \\
\hline CBS-QB3 & -231.78980 & - & - & -39.74480 & - & - \\
\hline \multirow[t]{3}{*}{ W1 } & -232.30029 & - & - & -39.84362 & - & - \\
\hline & \multicolumn{3}{|c|}{ TS• } & \multicolumn{3}{|c|}{$\mathrm{C}_{6} \mathrm{H}_{5}{ }^{\bullet}$} \\
\hline & $\mathbf{E}_{\mathbf{0}}$ & ZPVE & $\Delta \Delta \mathrm{H}$ & $\mathbf{E}_{\mathbf{0}}$ & ZPVE & $\Delta \Delta \mathbf{H}$ \\
\hline BP86/6-31G(d) & -272.05332 & 0.12531 & 0.13351 & -231.55272 & 0.08494 & 0.09014 \\
\hline $\mathrm{BP} 86 / 6-31+\mathrm{G}(\mathrm{d}, \mathrm{p})$ & -272.07774 & 0.12483 & 0.13400 & -231.56954 & 0.08465 & 0.09040 \\
\hline BLYP/6-31G(d) & -271.91886 & 0.12532 & 0.13397 & -231.45321 & 0.08487 & 0.09031 \\
\hline $\mathrm{BLYP} / 6-31+\mathrm{G}(\mathrm{d}, \mathrm{p})$ & -271.94807 & 0.12480 & 0.13345 & -231.47386 & 0.08453 & 0.09000 \\
\hline B3-P86/6-31G(d) & -272.98258 & 0.12954 & 0.13806 & -232.29683 & 0.08799 & 0.09330 \\
\hline B3-P86/6-31+G(d,p) & -273.00538 & 0.12902 & 0.13751 & -232.31224 & 0.08768 & 0.09301 \\
\hline B3-LYP/6-31G(d) & -272.06325 & 0.12909 & 0.13761 & -231.56128 & 0.08763 & 0.09294 \\
\hline B3-LYP/6-31G(d,p) & -272.07794 & 0.12880 & 0.13730 & -231.56922 & 0.08749 & 0.09281 \\
\hline B3-LYP/6-31+G(d,p) & -272.08995 & 0.12854 & 0.13703 & -231.57989 & 0.08728 & 0.09261 \\
\hline B3-LYP/6-311G(d,p) & -272.13686 & 0.12801 & 0.13655 & -231.61942 & 0.08703 & 0.09235 \\
\hline B3-PW91/6-31G(d) & -271.95881 & 0.12945 & 0.13793 & -231.47194 & 0.08788 & 0.09320 \\
\hline B3-PW91/6-31+G(d,p) & -271.98164 & 0.12888 & 0.13737 & -231.48737 & 0.08757 & 0.09291 \\
\hline MPW1K/6-31G(d) & -271.98418 & 0.13310 & 0.14143 & -231.49704 & 0.09051 & 0.09571 \\
\hline MPW1K/6-31+G(d,p) & -272.00648 & 0.13245 & 0.14079 & -231.51185 & 0.09016 & 0.09538 \\
\hline $\mathrm{BB} 1 \mathrm{~K} / 6-31 \mathrm{G}(\mathrm{d})$ & -271.90182 & 0.13236 & 0.14077 & -231.43996 & 0.08992 & 0.09517 \\
\hline $\mathrm{BB} 1 \mathrm{~K} / 6-31+\mathrm{G}(\mathrm{d}, \mathrm{p})$ & -271.92404 & 0.13170 & 0.14013 & -231.45492 & 0.08957 & 0.09483 \\
\hline MPWB1K/6-31G(d) & -271.89651 & 0.13270 & 0.14110 & -231.43575 & 0.09019 & 0.09542 \\
\hline MPWB1K/6-31+G(d,p) & -271.91947 & 0.13201 & 0.14043 & -231.45128 & 0.08982 & 0.09507 \\
\hline $\mathrm{BMK} / 6-31 \mathrm{G}(\mathrm{d})$ & -271.84759 & 0.13017 & 0.13858 & -231.38996 & 0.08823 & 0.09351 \\
\hline $\mathrm{BMK} / 6-31+\mathrm{G}(\mathrm{d}, \mathrm{p})$ & -271.86813 & 0.12941 & 0.13783 & -231.40426 & 0.08776 & 0.09307 \\
\hline $\mathrm{UHF} / 6-31 \mathrm{G}(\mathrm{d})$ & -270.21853 & 0.13532 & 0.14364 & -230.06507 & 0.09155 & 0.09676 \\
\hline $\mathrm{UHF} / 6-31+\mathrm{G}(\mathrm{d}, \mathrm{p})$ & -270.24256 & 0.13469 & 0.14209 & -230.08083 & 0.09129 & 0.09649 \\
\hline $\mathrm{RHF} / 6-31 \mathrm{G}(\mathrm{d})$ & -270.19775 & 0.13814 & 0.14622 & -230.05091 & 0.09410 & 0.09910 \\
\hline $\mathrm{RHF} / 6-31+\mathrm{G}(\mathrm{d}, \mathrm{p})$ & -270.22319 & 0.13726 & 0.14537 & -230.05091 & 0.09365 & 0.09866 \\
\hline MP2/6-31G(d) & -271.05791 & 0.13462 & 0.14288 & -230.74807 & 0.09250 & 0.09750 \\
\hline
\end{tabular}


TABLE S3: continued ...

\begin{tabular}{|c|c|c|c|c|c|c|}
\hline $\mathrm{MP} 2 / 6-31+\mathrm{G}(\mathrm{d}, \mathrm{p})$ & -271.14950 & 0.13431 & 0.14272 & -230.80299 & 0.09219 & 0.09730 \\
\hline QCISD/6-31G(d) & -271.14529 & 0.12956 & 0.13816 & -230.81748 & 0.08840 & 0.09370 \\
\hline QCISD/6-31+G(d,p) & -271.23469 & 0.12950 & 0.13745 & -230.87025 & 0.08834 & 0.09377 \\
\hline $\mathrm{CCSD} / 6-31 \mathrm{G}(\mathrm{d})$ & -271.14490 & $-b$ & $-b$ & -230.81750 & $-b$ & $-b$ \\
\hline $\mathrm{CCSD} / 6-31+\mathrm{G}(\mathrm{d}, \mathrm{p})$ & -271.23441 & $-b$ & $-b$ & -230.87028 & $-b$ & $-b$ \\
\hline $\mathrm{CCSD} / 6-311 \mathrm{G}(\mathrm{d}, \mathrm{p})$ & -271.30552 & 0.12826 & 0.13607 & -230.92627 & 0.08746 & 0.09284 \\
\hline $\begin{array}{l}\text { UMP2/ } \\
6-311+G(3 d f, 2 p)\end{array}$ & -271.38116 & $-b$ & $-b$ & -230.99398 & $-b$ & $-b$ \\
\hline $\begin{array}{l}\text { RMP2/ } \\
6-311+G(3 d f, 2 p)\end{array}$ & -271.42639 & $-b$ & $-b$ & -231.03337 & $-b$ & $-b$ \\
\hline $\begin{array}{l}\text { URCCSD/ } \\
6-311+G(3 d f, 2 p)\end{array}$ & -271.53724 & $-b$ & $-b$ & -231.12093 & $-b$ & $-b$ \\
\hline G3-RAD & -271.97565 & - & - & -231.48777 & - & - \\
\hline G3(MP2)-RAD & -271.74880 & - & - & -231.29166 & - & - \\
\hline CBS-QB3 & -271.63183 & - & - & -231.10830 & - & - \\
\hline \multirow[t]{3}{*}{$\mathrm{W} 1$} & - & - & - & -231.60954 & - & - \\
\hline & & $\mathrm{CH}_{4}$ & & & & \\
\hline & $\mathbf{E}_{0}$ & ZPVE & $\Delta \Delta \mathrm{H}$ & & & \\
\hline BP86/6-31G(d) & -40.51174 & 0.04408 & 0.04752 & & & \\
\hline $\mathrm{BP} 86 / 6-31+\mathrm{G}(\mathrm{d}, \mathrm{p})$ & -40.51900 & 0.04370 & 0.04790 & & & \\
\hline BLYP/6-31G(d) & -40.47913 & 0.04413 & 0.04794 & & & \\
\hline BLYP/6-31+G(d,p) & -40.48781 & 0.04371 & 0.04753 & & & \\
\hline B3-P86/6-31G(d) & -40.69999 & 0.04535 & 0.04916 & & & \\
\hline B3-P86/6-31+G(d,p) & -40.70667 & 0.04495 & 0.04876 & & & \\
\hline B3-LYP/6-31G(d) & -40.51839 & 0.04522 & 0.04903 & & & \\
\hline B3-LYP/6-31G(d,p) & -40.52402 & 0.04503 & 0.04884 & & & \\
\hline B3-LYP/6-31+G(d,p) & -40.52614 & 0.04479 & 0.04860 & & & \\
\hline B3-LYP/6-311G(d,p) & -40.53375 & 0.04459 & 0.04840 & & & \\
\hline B3-PW91/6-31G(d) & -40.50336 & 0.04530 & 0.04911 & & & \\
\hline B3-PW91/6-31+G(d,p) & -40.51007 & 0.04490 & 0.04871 & & & \\
\hline MPW1K/6-31G(d) & -40.50647 & 0.04642 & 0.05022 & & & \\
\hline MPW1K/6-31+G(d,p) & -40.51292 & 0.04599 & 0.04979 & & & \\
\hline $\mathrm{BB} 1 \mathrm{~K} / 6-31 \mathrm{G}(\mathrm{d})$ & -40.48134 & 0.04623 & 0.05004 & & & \\
\hline $\mathrm{BB} 1 \mathrm{~K} / 6-31+\mathrm{G}(\mathrm{d}, \mathrm{p})$ & -40.48792 & 0.04579 & 0.04960 & & & \\
\hline MPWB1K/6-31G(d) & -40.47930 & 0.04635 & 0.05015 & & & \\
\hline MPWB1K/6-31+G(d,p) & -40.48605 & 0.04638 & 0.05019 & & & \\
\hline $\mathrm{BMK} / 6-31 \mathrm{G}(\mathrm{d})$ & -40.47623 & 0.04530 & 0.04911 & & & \\
\hline $\mathrm{BMK} / 6-31+\mathrm{G}(\mathrm{d}, \mathrm{p})$ & -40.48160 & 0.04480 & 0.04861 & & & \\
\hline
\end{tabular}


TABLE S3: continued ...

\begin{tabular}{l|ccc}
\hline BMK/6-31+G(d,p) & -40.48160 & 0.04480 & 0.04861 \\
UHF/6-31G(d) & -40.19517 & 0.04781 & 0.05161 \\
UHF/6-31+G(d,p) & -40.20213 & 0.04727 & 0.05106 \\
RHF/6-31G(d) & -40.19517 & 0.04781 & 0.05161 \\
RHF/6-31+G(d,p) & -40.20213 & 0.04727 & 0.05106 \\
MP2/6-31G(d) & -40.33255 & 0.04635 & 0.05015 \\
MP2/6-31+G(d,p) & -40.36595 & 0.04625 & 0.05006 \\
QCISD/6-31G(d) & -40.35337 & 0.04561 & 0.04941 \\
QCISD/6-31+G(d,p) & -40.38743 & 0.04582 & 0.04962 \\
CCSD/6-31G(d) & -40.35310 & 0.04570 & 0.04950 \\
CCSD/6-31+G(d,p) & -40.38724 & 0.04589 & 0.04970 \\
CCSD/6-311G(d,p) & -40.40144 & 0.04510 & 0.04891 \\
UMP2/ & -40.40557 & $-b$ & $-b$ \\
6-311+G(3df,2p) & & & - \\
RMP2/ & -40.40557 & $-b$ & $-b$ \\
6-311+G(3df,2p) & & & $-b$ \\
URCCSD/ & -40.43452 & $-b$ & - \\
6-311+G(3df,2p) & -40.50683 & - & - \\
G3-RAD & -40.47458 & - & - \\
G3(MP2)-RAD & -40.4100 & - & - \\
CBS-QB3 & -40.52299 & - & - \\
W1 & & & - \\
\hline
\end{tabular}

${ }^{a}$ Corresponding to structures optimized at the specified level, unless otherwise noted.

${ }^{b}$ Frequencies were not calculated at this level, hence the ZPVE and TC are unavailable. 
TABLE S4: Wigner and Eckart Tunnelling Corrections Calculated for a Variety of Temperatures in the Interval 600-800 K

\begin{tabular}{lccccccccc}
\hline & & \multicolumn{7}{c}{ T $\mathbf{( K )}$} & \\
Wigner Corrections & $\mathbf{6 0 0}$ & $\mathbf{6 2 5}$ & $\mathbf{6 5 0}$ & $\mathbf{6 7 5}$ & $\mathbf{7 0 0}$ & $\mathbf{7 2 5}$ & $\mathbf{7 5 0}$ & $\mathbf{7 7 5}$ & $\mathbf{8 0 0}$ \\
\hline BP86/6-31G(d) & 1.41 & 1.38 & 1.35 & 1.32 & 1.30 & 1.28 & 1.26 & 1.25 & 1.23 \\
BP86/6-31+G(d,p) & 1.37 & 1.34 & 1.31 & 1.29 & 1.27 & 1.25 & 1.24 & 1.22 & 1.21 \\
BLYP/6-31G(d) & 1.49 & 1.45 & 1.42 & 1.39 & 1.36 & 1.33 & 1.31 & 1.29 & 1.27 \\
BLYP/6-31+G(d,p) & 1.45 & 1.42 & 1.39 & 1.36 & 1.33 & 1.31 & 1.29 & 1.27 & 1.26 \\
B3-P86/6-31G(d) & 1.55 & 1.50 & 1.47 & 1.43 & 1.40 & 1.37 & 1.35 & 1.33 & 1.31 \\
B3-P86/6-31+G(d,p) & 1.50 & 1.46 & 1.42 & 1.39 & 1.36 & 1.34 & 1.32 & 1.30 & 1.28 \\
B3-LYP/6-31G(d) & 1.62 & 1.57 & 1.53 & 1.49 & 1.45 & 1.42 & 1.39 & 1.37 & 1.35 \\
B3-LYP/6-31+G(d,p) & 1.57 & 1.53 & 1.49 & 1.45 & 1.42 & 1.39 & 1.37 & 1.34 & 1.32 \\
B3-PW91/6-31G(d) & 1.53 & 1.49 & 1.45 & 1.42 & 1.39 & 1.37 & 1.34 & 1.32 & 1.30 \\
B3-PW91/6-31+G(d,p) & 1.52 & 1.48 & 1.44 & 1.41 & 1.38 & 1.35 & 1.33 & 1.31 & 1.29 \\
MPW1K/6-31G(d) & 1.73 & 1.67 & 1.62 & 1.57 & 1.53 & 1.50 & 1.47 & 1.44 & 1.41 \\
MPW1K/6-31+G(d,p) & 1.67 & 1.62 & 1.57 & 1.53 & 1.49 & 1.46 & 1.43 & 1.40 & 1.38 \\
BB1K/6-31G(d) & 1.70 & 1.64 & 1.60 & 1.55 & 1.51 & 1.48 & 1.45 & 1.42 & 1.39 \\
BB1K/6-31+G(d,p) & 1.65 & 1.60 & 1.56 & 1.51 & 1.48 & 1.45 & 1.42 & 1.39 & 1.37 \\
MPWB1K/6-31G(d) & 1.71 & 1.65 & 1.60 & 1.56 & 1.52 & 1.48 & 1.45 & 1.42 & 1.40 \\
MPWB1K/6-31+G(d,p) & 1.66 & 1.61 & 1.56 & 1.52 & 1.48 & 1.45 & 1.42 & 1.39 & 1.37 \\
BMK/6-31G(d) & 1.70 & 1.65 & 1.60 & 1.55 & 1.52 & 1.48 & 1.45 & 1.42 & 1.39 \\
BMK/6-31+G(d,p) & 1.65 & 1.60 & 1.55 & 1.51 & 1.47 & 1.44 & 1.41 & 1.39 & 1.36 \\
\hline
\end{tabular}


TABLE S4: continued ...

\begin{tabular}{lccccccccc}
\hline & & & \multicolumn{7}{c}{ T (K) } \\
Eckart Corrections & $\mathbf{6 0 0}$ & $\mathbf{6 2 5}$ & $\mathbf{6 5 0}$ & $\mathbf{6 7 5}$ & $\mathbf{7 0 0}$ & $\mathbf{7 2 5}$ & $\mathbf{7 5 0}$ & $\mathbf{7 7 5}$ & $\mathbf{8 0 0}$ \\
\hline BP86/6-31G(d) & 1.56 & 1.51 & 1.47 & 1.43 & 1.40 & 1.37 & 1.35 & 1.32 & 1.30 \\
BP86/6-31+G(d,p) & 1.49 & 1.45 & 1.41 & 1.38 & 1.35 & 1.33 & 1.30 & 1.28 & 1.27 \\
BLYP/6-31G(d) & 1.70 & 1.63 & 1.58 & 1.53 & 1.49 & 1.45 & 1.42 & 1.39 & 1.36 \\
BLYP/6-31+G(d,p) & 1.64 & 1.58 & 1.53 & 1.48 & 1.44 & 1.41 & 1.38 & 1.36 & 1.33 \\
B3-P86/6-31G(d) & 1.80 & 1.72 & 1.66 & 1.60 & 1.55 & 1.51 & 1.47 & 1.44 & 1.41 \\
B3-P86/6-31+G(d,p) & 1.70 & 1.64 & 1.58 & 1.53 & 1.49 & 1.45 & 1.42 & 1.39 & 1.36 \\
B3-LYP/6-31G(d) & 1.95 & 1.85 & 1.77 & 1.70 & 1.64 & 1.59 & 1.54 & 1.50 & 1.47 \\
B3-LYP/6-31+G(d,p) & 1.86 & 1.77 & 1.70 & 1.64 & 1.58 & 1.54 & 1.50 & 1.46 & 1.43 \\
B3-PW91/6-31G(d) & 1.79 & 1.71 & 1.64 & 1.59 & 1.54 & 1.50 & 1.46 & 1.43 & 1.40 \\
B3-PW91/6-31+G(d,p) & 1.75 & 1.68 & 1.61 & 1.56 & 1.51 & 1.47 & 1.44 & 1.41 & 1.38 \\
MPW1K/6-31G(d) & 2.21 & 2.08 & 1.96 & 1.87 & 1.79 & 1.72 & 1.66 & 1.61 & 1.57 \\
MPW1K/6-31+G(d,p) & 2.07 & 1.95 & 1.86 & 1.78 & 1.71 & 1.65 & 1.59 & 1.55 & 1.51 \\
BB1K/6-31G(d) & 2.15 & 2.02 & 1.92 & 1.83 & 1.75 & 1.69 & 1.63 & 1.59 & 1.54 \\
BB1K/6-31+G(d,p) & 2.04 & 1.92 & 1.83 & 1.75 & 1.69 & 1.63 & 1.58 & 1.54 & 1.50 \\
MPWB1K/6-31G(d) & 2.16 & 2.03 & 1.92 & 1.84 & 1.76 & 1.70 & 1.64 & 1.59 & 1.55 \\
MPWB1K/6-31+G(d,p) & 2.04 & 1.93 & 1.83 & 1.76 & 1.69 & 1.63 & 1.58 & 1.54 & 1.50 \\
BMK/6-31G(d) & 2.15 & 2.02 & 1.92 & 1.83 & 1.76 & 1.69 & 1.64 & 1.59 & 1.54 \\
BMK/6-31+G(d,p) & 2.02 & 1.91 & 1.82 & 1.74 & 1.68 & 1.62 & 1.57 & 1.53 & 1.49 \\
\hline
\end{tabular}


TABLE S5: Effect of Level of Theory and Internal Rotation on Activation Energies $E_{a}(\mathrm{~kJ}$ $\left.\mathrm{mol}^{-1}\right)$ and Pre-Exponential Factors $A\left(\mathrm{~m}^{3} \mathrm{~mol}^{-1} \mathrm{~s}^{-1}\right)$ for the Benzene Abstraction Reaction in the Temperature Interval 600-800 K (Eckart Tunnelling Corrections Included). Also Shown is the Frequency $\left(v_{m}, \mathrm{~cm}^{-1}\right)$ for the Internal Rotation of the Methyl Group in the TS

\begin{tabular}{lccccc}
\hline \multicolumn{1}{c}{ Level } & \multicolumn{2}{c}{$\boldsymbol{E}_{\boldsymbol{a}}$} & & \multicolumn{2}{c}{$\boldsymbol{A}$} \\
\hline BP86/6-31G(d) & HO & $\boldsymbol{H O} / \boldsymbol{F R}$ & $\boldsymbol{v}_{\boldsymbol{m}}$ & $\boldsymbol{H O}$ & $\boldsymbol{H O} / \boldsymbol{F R}$ \\
BP86/6-31+G(d,p) & 46.4 & 43.5 & 19.4 & $2.04 \times 10^{8}$ & $2.84 \times 10^{7}$ \\
BLYP/6-31G(d) & 51.2 & 48.3 & 28.5 & $1.57 \times 10^{8}$ & $3.20 \times 10^{7}$ \\
BLYP/6-31+G(d,p) & 58.6 & 50.1 & 22.4 & $4.37 \times 10^{8}$ & $2.59 \times 10^{7}$ \\
B3-P86/6-31G(d) & 62.6 & 56.3 & 25.0 & $1.31 \times 10^{8}$ & $2.72 \times 10^{7}$ \\
B3-P86/6-31+G(d,p) & 52.9 & 55.7 & 6.9 & $1.61 \times 10^{8}$ & $2.14 \times 10^{7}$ \\
B3-LYP/6-31G(d) & 59.2 & 59.7 & 26.9 & $1.52 \times 10^{8}$ & $2.51 \times 10^{7}$ \\
B3-LYP/6-31+G(d,p) & 64.4 & 61.5 & 3.4 & $7.72 \times 10^{8}$ & $1.89 \times 10^{7}$ \\
B3-PW91/6-31G(d) & 69.9 & 67.1 & 23.4 & $1.31 \times 10^{8}$ & $2.18 \times 10^{7}$ \\
B3-PW91/6-31+G(d,p) & 64.3 & 61.4 & 24.3 & $1.16 \times 10^{8}$ & $1.99 \times 10^{7}$ \\
MPW1K/6-31G(d) & 68.2 & 65.3 & 23.2 & $1.40 \times 10^{8}$ & $2.30 \times 10^{7}$ \\
MPW1K/6-31+G(d,p) & 74.7 & 71.8 & 26.0 & $8.21 \times 10^{7}$ & $1.51 \times 10^{7}$ \\
BB1K/6-31G(d) & 78.4 & 75.5 & 26.6 & $9.82 \times 10^{7}$ & $1.85 \times 10^{7}$ \\
BB1K/6-31+G(d,p) & 68.0 & 66.1 & 15.8 & $1.19 \times 10^{8}$ & $1.87 \times 10^{7}$ \\
MPWB1K/6-31G(d) & 72.3 & 71.0 & 16.3 & $1.38 \times 10^{8}$ & $2.35 \times 10^{7}$ \\
MPWB1K/6-31+G(d,p) & 69.0 & 65.1 & 20.2 & $1.67 \times 10^{8}$ & $1.69 \times 10^{7}$ \\
BMK/6-31G(d) & 73.9 & 70.2 & 20.5 & $2.05 \times 10^{8}$ & $2.16 \times 10^{7}$ \\
BMK/6-31+G(d,p) & 69.5 & 66.6 & 43.0 & $6.25 \times 10^{7}$ & $1.91 \times 10^{7}$ \\
\hline
\end{tabular}


TABLE S6: Summary of Theoretical Rate Constants ( $\ln \mathbf{k}_{\text {theory }}$ ) and $f_{k}$ Values (Calculated Using the Formula $\left.f_{k}=k_{\text {theory }} / k_{\text {expt }}\right)$ for the Forward Reaction $\left(\mathrm{C}_{6} \mathrm{H}_{6}+\cdot \mathrm{CH}_{3} \rightarrow \cdot \mathrm{C}_{6} \mathrm{H}_{5}+\right.$ $\left.\mathrm{CH}_{4}\right)$ at a Variety Temperature in the Interval 600-800 K. Basis Set Legend: $1=6-31 \mathrm{G}(\mathrm{d})$; $2=6-31+G(d, p) ;$ and $3=6-311+G(3 d f, 2 p)$

\begin{tabular}{|c|c|c|c|c|c|c|c|c|c|c|c|}
\hline 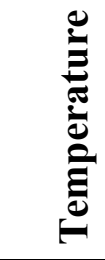 & $\begin{array}{l}\bar{D} \\
\infty \\
\stackrel{\infty}{\infty}\end{array}$ & 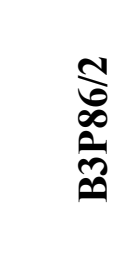 & $\underset{\tilde{\infty}}{\bar{\partial}}$ & $\frac{N}{\stackrel{N}{\frac{N}{n}}}$ & $\frac{\vec{a}}{\hat{a}}$ & $\begin{array}{l}\stackrel{N}{a} \\
\hat{a} \\
\hat{a} \\
\hat{a}\end{array}$ & $\frac{1}{\sum}$ & 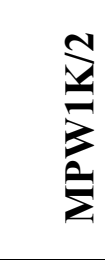 & $\frac{\vec{D}}{\oplus \theta}$ & $\frac{\vec{a}}{\vec{m}}$ & 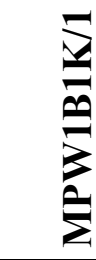 \\
\hline \multicolumn{12}{|c|}{ Theoretical rate constants (ln $\left.\mathbf{k}_{\text {theory }}\right)$} \\
\hline 600 & 5.71 & 5.07 & 4.43 & 3.45 & 4.50 & 3.86 & 2.14 & 1.60 & 3.49 & 2.74 & 3.59 \\
\hline 650 & 6.57 & 5.99 & 5.38 & 4.48 & 5.45 & 4.87 & 3.24 & 2.76 & 4.51 & 3.84 & 4.60 \\
\hline 700 & 7.31 & 6.78 & 6.19 & 5.37 & 6.26 & 5.73 & 4.19 & 3.76 & 5.39 & 4.77 & 5.46 \\
\hline 750 & 7.95 & 7.46 & 6.89 & 6.14 & 6.96 & 6.48 & 5.02 & 4.63 & 6.14 & 5.59 & 6.20 \\
\hline 760 & 8.06 & 7.59 & 7.02 & 6.28 & 7.09 & 6.62 & 5.17 & 4.79 & 6.28 & 5.74 & 6.34 \\
\hline 770 & 8.18 & 7.71 & 7.15 & 6.42 & 7.22 & 6.75 & 5.32 & 4.94 & 6.42 & 5.88 & 6.47 \\
\hline 780 & 8.29 & 7.83 & 7.27 & 6.55 & 7.34 & 6.88 & 5.46 & 5.09 & 6.55 & 6.02 & 6.60 \\
\hline 790 & 8.40 & 7.95 & 7.39 & 6.68 & 7.46 & 7.01 & 5.60 & 5.24 & 6.68 & 6.16 & 6.73 \\
\hline 800 & 8.50 & 8.06 & 7.51 & 6.81 & 7.58 & 7.13 & 5.74 & 5.38 & 6.81 & 6.30 & 6.86 \\
\hline
\end{tabular}

$f_{k}$ values comparing $k_{\text {theory }}$ with the $k_{\text {expt }}$ values of Krech and Price [26]

$\begin{array}{cccccccccccc}750 & 23.00 & 14.20 & 8.01 & 3.77 & 8.57 & 5.30 & 1.23 & 0.83 & 3.79 & 2.17 & 4.02 \\ 760 & 23.83 & 14.84 & 8.41 & 4.00 & 8.99 & 5.61 & 1.32 & 0.90 & 4.02 & 2.32 & 4.25 \\ 770 & 24.67 & 15.49 & 8.80 & 4.23 & 9.42 & 5.92 & 1.41 & 0.97 & 4.25 & 2.48 & 4.49 \\ 780 & 25.51 & 16.15 & 9.21 & 4.48 & 9.85 & 6.24 & 1.50 & 1.04 & 4.48 & 2.65 & 4.73 \\ 790 & 26.36 & 16.82 & 9.63 & 4.73 & 10.29 & 6.57 & 1.60 & 1.12 & 4.73 & 2.82 & 4.98 \\ 800 & 27.22 & 17.50 & 10.05 & 5.00 & 10.74 & 6.91 & 1.71 & 1.20 & 4.98 & 3.00 & 5.23 \\ <\mathbf{f}_{\mathbf{k}}> & \mathbf{2 5 . 1 0} & \mathbf{1 5 . 8 3} & \mathbf{9 . 0 2} & \mathbf{4 . 3 7} & \mathbf{9 . 6 4} & \mathbf{6 . 0 9} & \mathbf{1 . 4 6} & \mathbf{1 . 0 1} & \mathbf{4 . 3 7} & \mathbf{2 . 5 7} & \mathbf{4 . 6 2}\end{array}$

$\mathbf{f}_{\mathbf{k}}$ values comparing $\mathbf{k}_{\text {theory }}$ with the $\mathbf{k}_{\text {expt }}$ values of Zhang et al. [27]

\begin{tabular}{llllllllllll}
650 & 41.59 & 23.27 & 12.56 & 5.14 & 13.47 & 7.57 & 1.49 & 0.92 & 5.30 & 2.69 & 5.77 \\
700 & 37.76 & 22.27 & 12.31 & 5.43 & 13.19 & 7.80 & 1.68 & 1.09 & 5.53 & 2.99 & 5.93 \\
750 & 34.73 & 21.45 & 12.10 & 5.69 & 12.95 & 8.01 & 1.85 & 1.25 & 5.73 & 3.28 & 6.07 \\
770 & 33.69 & 21.15 & 12.02 & 5.78 & 12.86 & 8.08 & 1.92 & 1.32 & 5.80 & 3.39 & 6.13 \\
$\left.<\mathbf{f}_{\mathbf{k}}\right\rangle$ & $\mathbf{3 7 . 3 1}$ & $\mathbf{2 2 . 1 4}$ & $\mathbf{1 2 . 2 8}$ & $\mathbf{5 . 4 7}$ & $\mathbf{1 3 . 1 5}$ & $\mathbf{7 . 8 3}$ & $\mathbf{1 . 7 1}$ & $\mathbf{1 . 1 2}$ & $\mathbf{5 . 5 6}$ & $\mathbf{3 . 0 5}$ & $\mathbf{5 . 9 6}$ \\
\hline
\end{tabular}


TABLE S6: continued ...

\begin{tabular}{|c|c|c|c|c|c|c|c|c|c|c|c|}
\hline 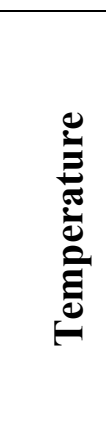 & $\underset{2}{\stackrel{N}{2}}$ & $\sum$ & $\underset{n}{\stackrel{N}{V}}$ & 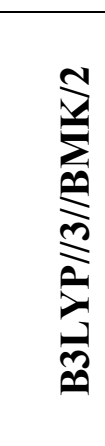 & 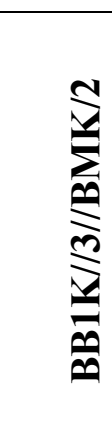 & 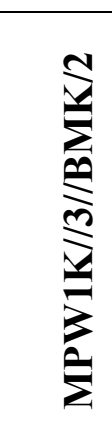 & $\underset{\sum_{\infty}^{\infty}}{\stackrel{N}{N}}$ & 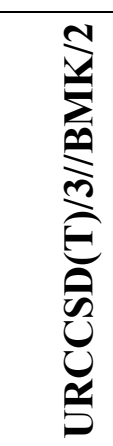 & $\begin{array}{l}\tilde{0} \\
\hat{0} \\
\dot{1} \\
\tilde{0} \\
0\end{array}$ & 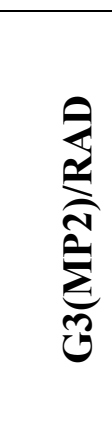 & 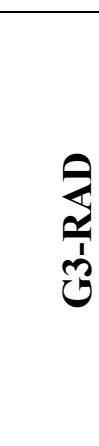 \\
\hline \multicolumn{12}{|c|}{ Theoretical rate constants (In $\mathbf{k}_{\text {theory }}$ ) } \\
\hline 600 & 2.82 & 3.42 & 2.69 & 2.99 & 2.14 & 1.54 & 1.94 & 1.68 & 0.64 & 1.34 & 1.60 \\
\hline 650 & 3.90 & 4.44 & 3.78 & 4.06 & 3.28 & 2.73 & 3.10 & 2.86 & 1.90 & 2.53 & 2.77 \\
\hline 700 & 4.83 & 5.32 & 4.72 & 4.98 & 4.26 & 3.74 & 4.09 & 3.86 & 2.97 & 3.54 & 3.77 \\
\hline 750 & 5.63 & 6.09 & 5.54 & 5.78 & 5.10 & 4.62 & 4.94 & 4.73 & 3.91 & 4.42 & 4.63 \\
\hline 760 & 5.78 & 6.23 & 5.69 & 5.92 & 5.26 & 4.78 & 5.10 & 4.89 & 4.08 & 4.59 & 4.79 \\
\hline 770 & 5.92 & 6.36 & 5.83 & 6.07 & 5.41 & 4.94 & 5.25 & 5.05 & 4.25 & 4.74 & 4.95 \\
\hline 780 & 6.06 & 6.50 & 5.97 & 6.21 & 5.56 & 5.10 & 5.40 & 5.20 & 4.41 & 4.90 & 5.10 \\
\hline 790 & 6.20 & 6.63 & 6.11 & 6.34 & 5.70 & 5.25 & 5.55 & 5.35 & 4.57 & 5.05 & 5.25 \\
\hline 800 & 6.33 & 6.75 & 6.25 & 6.47 & 5.84 & 5.39 & 5.69 & 5.50 & 4.72 & 5.19 & 5.39 \\
\hline \multicolumn{12}{|c|}{$f_{k}$ values comparing $k_{\text {theory }}$ with the $k_{\text {expt }}$ values of Krech and Price [26] } \\
\hline 750 & 2.27 & 3.57 & 2.06 & 2.63 & 1.34 & 0.83 & 1.14 & 0.93 & 0.40 & 0.68 & 0.84 \\
\hline 760 & 2.42 & 3.79 & 2.21 & 2.80 & 1.44 & 0.90 & 1.23 & 1.00 & 0.44 & 0.73 & 0.90 \\
\hline 770 & 2.59 & 4.01 & 2.36 & 2.98 & 1.55 & 0.97 & 1.32 & 1.08 & 0.48 & 0.79 & 0.97 \\
\hline 780 & 2.75 & 4.24 & 2.52 & 3.17 & 1.66 & 1.05 & 1.42 & 1.16 & 0.53 & 0.86 & 1.05 \\
\hline 790 & 2.93 & 4.48 & 2.68 & 3.37 & 1.78 & 1.13 & 1.53 & 1.25 & 0.57 & 0.92 & 1.13 \\
\hline 800 & 3.11 & 4.72 & 2.85 & 3.57 & 1.90 & 1.21 & 1.63 & 1.34 & 0.62 & 0.99 & 1.21 \\
\hline$<\mathbf{f}_{\mathbf{k}}>$ & 2.68 & 4.14 & 2.45 & 3.09 & 1.61 & 1.01 & 1.38 & 1.13 & 0.51 & 0.83 & 1.02 \\
\hline \multicolumn{12}{|c|}{$\mathbf{f}_{\mathbf{k}}$ values comparing $k_{\text {theory }}$ with the $k_{\text {expt }}$ values of Zhang et al. [27] } \\
\hline 650 & 2.87 & 4.94 & 2.55 & 3.37 & 1.55 & 0.89 & 1.29 & 1.01 & 0.39 & 0.73 & 0.92 \\
\hline 700 & 3.16 & 5.18 & 2.84 & 3.68 & 1.79 & 1.07 & 1.50 & 1.20 & 0.49 & 0.87 & 1.09 \\
\hline 750 & 3.43 & 5.40 & 3.12 & 3.96 & 2.02 & 1.25 & 1.72 & 1.40 & 0.61 & 1.02 & 1.26 \\
\hline 760 & 3.48 & 5.44 & 3.17 & 4.02 & 2.07 & 1.29 & 1.77 & 1.44 & 0.63 & 1.05 & 1.30 \\
\hline 770 & 3.53 & 5.48 & 3.22 & 4.08 & 2.11 & 1.32 & 1.81 & 1.48 & 0.66 & 1.08 & 1.33 \\
\hline$<\mathbf{f}_{\mathbf{k}}>$ & 3.21 & 5.22 & 2.89 & 3.73 & 1.83 & 1.10 & 1.55 & 1.24 & 0.52 & 0.90 & 1.13 \\
\hline
\end{tabular}


TABLE S7: Summary of Theoretical Rate Constants $\left(\ln k_{\text {theory }}\right)$ and $f_{k}$ Values (Calculated Using the Formula $\left.f_{k}=k_{\text {theory }} / k_{\text {expt }}\right)$ for the Reverse Reaction $\left(\cdot \mathrm{C}_{6} \mathbf{H}_{5}+\mathrm{CH}_{4} \rightarrow \mathrm{C}_{6} \mathrm{H}_{6}+\cdot \mathrm{CH}_{3}\right)$ in the Temperature Interval 600-800 K. Basis Set Legend: $1=6-31 G(d) ; 2=6-31+G(d, p)$; and $3=6-311+G(3 d f, 2 p)$

\begin{tabular}{|c|c|c|c|c|c|c|c|c|c|c|c|}
\hline 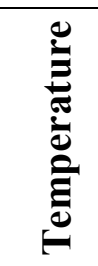 & $\begin{array}{l}\bar{\delta} \\
\infty \\
\stackrel{\infty}{\infty} \\
\stackrel{0}{0}\end{array}$ & $\begin{array}{l}\hat{\sigma} \\
\infty \\
\infty \\
\hat{\infty} \\
\infty\end{array}$ & $\underset{\ddot{\theta}}{\vec{\infty}}$ & $\underset{\tilde{n}}{\stackrel{v}{a}}$ & $\stackrel{\vec{a}}{\hat{n}}$ & $\begin{array}{l}\stackrel{N}{a} \\
\hat{a} \\
\hat{n}\end{array}$ & $\frac{\vec{v}}{\sum}$ & $\frac{N}{\sum}$ & $\underset{\theta}{\Delta}$ & $\frac{\stackrel{N}{\underline{n}}}{\underline{n}}$ & $\frac{\vec{D}}{\vec{\theta}}$ \\
\hline \multicolumn{12}{|c|}{ Theoretical rate constants (ln $\left.k_{\text {theory }}\right)$} \\
\hline 600 & 9.28 & 9.53 & 8.17 & 8.15 & 8.05 & 8.30 & 6.73 & 7.14 & 6.71 & 6.95 & 7.27 \\
\hline 610 & 9.40 & 9.64 & 8.30 & 8.28 & 8.18 & 8.43 & 6.88 & 7.28 & 6.86 & 7.10 & 7.41 \\
\hline 620 & 9.51 & 9.74 & 8.43 & 8.41 & 8.31 & 8.55 & 7.02 & 7.42 & 7.01 & 7.24 & 7.55 \\
\hline 630 & 9.62 & 9.84 & 8.55 & 8.53 & 8.43 & 8.67 & 7.16 & 7.56 & 7.16 & 7.38 & 7.69 \\
\hline 640 & 9.72 & 9.94 & 8.67 & 8.65 & 8.56 & 8.79 & 7.30 & 7.69 & 7.30 & 7.52 & 7.82 \\
\hline 650 & 9.82 & 10.04 & 8.78 & 8.76 & 8.67 & 8.90 & 7.43 & 7.82 & 7.43 & 7.65 & 7.95 \\
\hline 660 & 9.92 & 10.14 & 8.89 & 8.88 & 8.79 & 9.01 & 7.56 & 7.94 & 7.56 & 7.78 & 8.07 \\
\hline 670 & 10.01 & 10.23 & 9.00 & 8.98 & 8.90 & 9.12 & 7.69 & 8.06 & 7.69 & 7.90 & 8.19 \\
\hline 680 & 10.10 & 10.32 & 9.11 & 9.09 & 9.00 & 9.22 & 7.81 & 8.18 & 7.81 & 8.02 & 8.31 \\
\hline 690 & 10.19 & 10.40 & 9.21 & 9.19 & 9.11 & 9.32 & 7.93 & 8.29 & 7.93 & 8.14 & 8.42 \\
\hline 700 & 10.28 & 10.48 & 9.31 & 9.29 & 9.21 & 9.42 & 8.04 & 8.40 & 8.05 & .26 & 8.53 \\
\hline 710 & 10.36 & 1 & 9.40 & 9. & 9.31 & 9.52 & 8.15 & 51 & 8.16 & 7 & 8.64 \\
\hline 720 & 10.44 & 10.64 & 9.50 & 9.48 & 9.40 & 9.61 & 8.26 & 8.61 & 8.27 & 8.47 & 8.74 \\
\hline 730 & 10.52 & 10.72 & 9.59 & 9.57 & 9.50 & 9.70 & 8.37 & 8.71 & 8.38 & 8.58 & 8.85 \\
\hline 740 & 10.60 & 10.80 & 9.68 & 9.66 & 9.59 & 9.79 & 8.47 & & 49 & & 8.94 \\
\hline 750 & 10.68 & 10.87 & 9.76 & 9.74 & 9.68 & 9.87 & 8.57 & 8.90 & 8.59 & 8.78 & 9.04 \\
\hline 760 & 10.75 & 10.94 & 9.85 & 9.82 & 9.76 & 9.95 & 8.67 & 9.00 & 8.69 & 8.87 & 9.13 \\
\hline 770 & 10.82 & 11.01 & 9.93 & 9.91 & 9.84 & 10.03 & 8.76 & 9.09 & 8.78 & 8.97 & 9.22 \\
\hline 780 & 10.89 & 11.07 & 10.01 & 9.99 & 9.93 & & 35 & 8 & .88 & 6 & 9.31 \\
\hline 790 & 10.96 & & 10.08 & & & & & .26 & 97 & & 9.40 \\
\hline 800 & 11.02 & 11.20 & 10.16 & 10.14 & 8 & 10. & 3 & .35 & 06 & & 9.48 \\
\hline \multicolumn{12}{|c|}{$f_{k}$ values comparing $k_{\text {theory }}$ with the $k_{\text {expt }}$ values of Tokmakov et al. [22] } \\
\hline 600 & 55.04 & 70.01 & 18.06 & 17.77 & 15.97 & 20.55 & 4.26 & 6.42 & 4.20 & 5.31 & 7.33 \\
\hline 610 & 52.07 & 65.97 & 17.37 & 17.08 & 15.41 & 19.74 & 4.18 & 6.27 & 4.13 & 5.21 & 7.15 \\
\hline 620 & 49.36 & 62.29 & 16.72 & 16.44 & 14.88 & 18.97 & 4.11 & 6.12 & 4.07 & 5.11 & 6.99 \\
\hline 630 & 46.86 & 58.92 & 16.12 & 15.85 & 14.39 & 18.27 & 4.04 & 5.98 & 4.01 & 5.02 & 6.83 \\
\hline 640 & 44.56 & 55.83 & 15.56 & 15.29 & 13.92 & 17.61 & 3.97 & 5.85 & 3.95 & 4.94 & 6.68 \\
\hline 650 & 42.44 & 52.99 & 15.04 & 14.77 & 13.49 & 16.99 & 3.91 & 5.73 & 3.90 & 4.85 & 6.54 \\
\hline 660 & 40.48 & 50.38 & 14.55 & 14.29 & 13.08 & 16.41 & 3.85 & 5.61 & 3.85 & 4.77 & 6.41 \\
\hline 670 & 38.67 & 47.96 & 14.09 & 13.83 & 12.70 & 15.87 & 3.79 & 5.49 & 3.80 & 4.70 & 6.28 \\
\hline 680 & 36.98 & 45.73 & 13.65 & 13.40 & 12.33 & 15.36 & 3.73 & 5.39 & 3.75 & 4.63 & 6.16 \\
\hline 690 & 35.42 & 43.66 & 13.24 & 13.00 & 11.99 & 14.88 & 3.68 & 5.29 & 3.70 & 4.56 & 6.04 \\
\hline
\end{tabular}


TABLE S7: continued ...

$\begin{array}{rrrrrrrrrrrr}700 & 33.96 & 41.74 & 12.86 & 12.61 & 11.67 & 14.43 & 3.63 & 5.19 & 3.66 & 4.49 & 5.93 \\ 710 & 32.61 & 39.96 & 12.49 & 12.25 & 11.36 & 14.00 & 3.58 & 5.10 & 3.62 & 4.43 & 5.83 \\ 720 & 31.34 & 38.29 & 12.15 & 11.91 & 11.08 & 13.60 & 3.54 & 5.01 & 3.58 & 4.37 & 5.73 \\ 730 & 30.15 & 36.74 & 11.83 & 11.59 & 10.80 & 13.22 & 3.49 & 4.92 & 3.54 & 4.31 & 5.63 \\ 740 & 29.04 & 35.30 & 11.52 & 11.29 & 10.54 & 12.86 & 3.45 & 4.84 & 3.50 & 4.25 & 5.54 \\ 750 & 28.00 & 33.94 & 11.22 & 11.00 & 10.29 & 12.52 & 3.41 & 4.76 & 3.47 & 4.20 & 5.45 \\ 760 & 27.02 & 32.67 & 10.95 & 10.73 & 10.06 & 12.20 & 3.37 & 4.69 & 3.43 & 4.15 & 5.37 \\ 770 & 26.10 & 31.48 & 10.68 & 10.47 & 9.83 & 11.89 & 3.33 & 4.62 & 3.40 & 4.10 & 5.29 \\ 780 & 25.24 & 30.36 & 10.43 & 10.22 & 9.62 & 11.60 & 3.29 & 4.55 & 3.37 & 4.05 & 5.21 \\ 790 & 24.42 & 29.31 & 10.19 & 9.98 & 9.42 & 11.33 & 3.26 & 4.48 & 3.34 & 4.00 & 5.14 \\ 800 & 23.65 & 28.32 & 9.97 & 9.76 & 9.22 & 11.06 & 3.22 & 4.42 & 3.31 & 3.96 & 5.07 \\ <\mathbf{f}_{\mathbf{k}}> & \mathbf{3 5 . 8 8} & \mathbf{4 4 . 3 8} & \mathbf{1 3 . 2 7} & \mathbf{1 3 . 0 3} & \mathbf{1 2 . 0 0} & \mathbf{1 4 . 9 2} & \mathbf{3 . 6 7} & \mathbf{5 . 2 7} & \mathbf{3 . 6 9} & \mathbf{4 . 5 4} & \mathbf{6 . 0 3}\end{array}$

$f_{k}$ values comparing $k_{\text {theory }}$ with the $k_{\text {expt }}$ values of Heckmann et al. [28]

$\begin{array}{cccccccccccc}600 & 7.34 & 9.34 & 2.41 & 2.37 & 2.13 & 2.74 & 0.57 & 0.86 & 0.56 & 0.71 & 0.98 \\ 610 & 7.31 & 9.26 & 2.44 & 2.40 & 2.16 & 2.77 & 0.59 & 0.88 & 0.58 & 0.73 & 1.00 \\ 620 & 7.28 & 9.19 & 2.47 & 2.43 & 2.19 & 2.80 & 0.61 & 0.90 & 0.60 & 0.75 & 1.03 \\ 630 & 7.25 & 9.12 & 2.49 & 2.45 & 2.23 & 2.83 & 0.62 & 0.93 & 0.62 & 0.78 & 1.06 \\ 640 & 7.22 & 9.05 & 2.52 & 2.48 & 2.26 & 2.85 & 0.64 & 0.95 & 0.64 & 0.80 & 1.08 \\ 650 & 7.20 & 8.98 & 2.55 & 2.50 & 2.29 & 2.88 & 0.66 & 0.97 & 0.66 & 0.82 & 1.11 \\ 660 & 7.17 & 8.92 & 2.58 & 2.53 & 2.32 & 2.91 & 0.68 & 0.99 & 0.68 & 0.85 & 1.13 \\ 670 & 7.15 & 8.86 & 2.60 & 2.56 & 2.35 & 2.93 & 0.70 & 1.02 & 0.70 & 0.87 & 1.16 \\ 680 & 7.12 & 8.80 & 2.63 & 2.58 & 2.37 & 2.96 & 0.72 & 1.04 & 0.72 & 0.89 & 1.19 \\ 690 & 7.10 & 8.75 & 2.65 & 2.60 & 2.40 & 2.98 & 0.74 & 1.06 & 0.74 & 0.91 & 1.21 \\ 700 & 7.08 & 8.70 & 2.68 & 2.63 & 2.43 & 3.01 & 0.76 & 1.08 & 0.76 & 0.94 & 1.24 \\ 710 & 7.05 & 8.64 & 2.70 & 2.65 & 2.46 & 3.03 & 0.78 & 1.10 & 0.78 & 0.96 & 1.26 \\ 720 & 7.03 & 8.59 & 2.73 & 2.67 & 2.49 & 3.05 & 0.79 & 1.12 & 0.80 & 0.98 & 1.29 \\ 730 & 7.01 & 8.54 & 2.75 & 2.70 & 2.51 & 3.07 & 0.81 & 1.14 & 0.82 & 1.00 & 1.31 \\ 740 & 6.99 & 8.50 & 2.77 & 2.72 & 2.54 & 3.10 & 0.83 & 1.17 & 0.84 & 1.02 & 1.33 \\ 750 & 6.97 & 8.45 & 2.80 & 2.74 & 2.56 & 3.12 & 0.85 & 1.19 & 0.86 & 1.05 & 1.36 \\ 760 & 6.95 & 8.41 & 2.82 & 2.76 & 2.59 & 3.14 & 0.87 & 1.21 & 0.88 & 1.07 & 1.38 \\ 770 & 6.94 & 8.36 & 2.84 & 2.78 & 2.61 & 3.16 & 0.88 & 1.23 & 0.90 & 1.09 & 1.41 \\ 780 & 6.92 & 8.32 & 2.86 & 2.80 & 2.64 & 3.18 & 0.90 & 1.25 & 0.92 & 1.11 & 1.43 \\ 790 & 6.90 & 8.28 & 2.88 & 2.82 & 2.66 & 3.20 & 0.92 & 1.27 & 0.94 & 1.13 & 1.45 \\ 800 & 6.88 & 8.24 & 2.90 & 2.84 & 2.68 & 3.22 & 0.94 & 1.29 & 0.96 & 1.15 & 1.47 \\ <\mathbf{f}> & \mathbf{7 . 0 9} & \mathbf{8 . 7 3} & \mathbf{2 . 6 7} & \mathbf{2 . 6 2} & \mathbf{2 . 4 2} & \mathbf{3 . 0 0} & \mathbf{0 . 7 6} & \mathbf{1 . 0 8} & \mathbf{0 . 7 6} & \mathbf{0 . 9 3} & \mathbf{1 . 2 3}\end{array}$

$f_{k}$ values comparing $k_{\text {theory }}$ with the $k_{\text {expt }}$ values of Duncan et al. [29]

$\begin{array}{llllllllllll}600 & 149.28 & 189.88 & 48.97 & 48.18 & 43.32 & 55.75 & 11.55 & 17.42 & 11.38 & 14.40 & 19.87 \\ 610 & 143.65 & 181.99 & 47.91 & 47.12 & 42.50 & 54.44 & 11.53 & 17.29 & 11.39 & 14.37 & 19.73 \\ 620 & 138.40 & 174.68 & 46.90 & 46.11 & 41.72 & 53.21 & 11.52 & 17.17 & 11.41 & 14.34 & 19.59 \\ 630 & 133.51 & 167.88 & 45.94 & 45.16 & 40.99 & 52.04 & 11.50 & 17.04 & 11.42 & 14.31 & 19.46\end{array}$


TABLE S7: continued ...

$\begin{array}{rrrrrrrrrrrr}640 & 128.93 & 161.54 & 45.03 & 44.25 & 40.28 & 50.94 & 11.49 & 16.93 & 11.44 & 14.28 & 19.33 \\ 650 & 124.64 & 155.63 & 44.17 & 43.39 & 39.61 & 49.89 & 11.48 & 16.82 & 11.45 & 14.25 & 19.21 \\ 660 & 120.62 & 150.10 & 43.35 & 42.57 & 38.97 & 48.89 & 11.46 & 16.71 & 11.46 & 14.22 & 19.09 \\ 670 & 116.84 & 144.92 & 42.56 & 41.79 & 38.36 & 47.94 & 11.45 & 16.60 & 11.47 & 14.20 & 18.98 \\ 680 & 113.29 & 140.07 & 41.82 & 41.05 & 37.78 & 47.04 & 11.44 & 16.50 & 11.49 & 14.17 & 18.87 \\ 690 & 109.94 & 135.52 & 41.10 & 40.34 & 37.22 & 46.18 & 11.43 & 16.41 & 11.50 & 14.14 & 18.76 \\ 700 & 106.78 & 131.23 & 40.42 & 39.66 & 36.69 & 45.36 & 11.42 & 16.31 & 11.51 & 14.12 & 18.66 \\ 710 & 103.80 & 127.20 & 39.77 & 39.01 & 36.18 & 44.58 & 11.41 & 16.22 & 11.52 & 14.10 & 18.56 \\ 720 & 100.98 & 123.40 & 39.15 & 38.39 & 35.69 & 43.83 & 11.39 & 16.13 & 11.53 & 14.07 & 18.46 \\ 730 & 98.31 & 119.81 & 38.56 & 37.80 & 35.22 & 43.11 & 11.38 & 16.05 & 11.54 & 14.05 & 18.37 \\ 740 & 95.79 & 116.41 & 37.99 & 37.23 & 34.77 & 42.43 & 11.37 & 15.97 & 11.55 & 14.03 & 18.28 \\ 750 & 93.39 & 113.20 & 37.44 & 36.69 & 34.33 & 41.77 & 11.36 & 15.89 & 11.56 & 14.01 & 18.19 \\ 760 & 91.11 & 110.17 & 36.91 & 36.16 & 33.91 & 41.14 & 11.35 & 15.81 & 11.57 & 13.99 & 18.11 \\ 770 & 88.95 & 107.28 & 36.41 & 35.66 & 33.51 & 40.53 & 11.35 & 15.74 & 11.58 & 13.97 & 18.03 \\ 780 & 86.89 & 104.55 & 35.92 & 35.18 & 33.13 & 39.95 & 11.34 & 15.66 & 11.59 & 13.95 & 17.95 \\ 790 & 84.92 & 101.95 & 35.46 & 34.72 & 32.75 & 39.40 & 11.33 & 15.59 & 11.60 & 13.93 & 17.87 \\ 800 & 83.05 & 99.48 & 35.01 & 34.27 & 32.39 & 38.86 & 11.32 & 15.53 & 11.61 & 13.91 & 17.80 \\ <\mathbf{f}_{\mathbf{k}}> & \mathbf{1 0 8 . 1 9} & \mathbf{1 3 3 . 3 5} & \mathbf{4 0 . 5 9} & \mathbf{3 9 . 8 3} & \mathbf{3 6 . 8 0} & \mathbf{4 5 . 5 8} & \mathbf{1 1 . 4 2} & \mathbf{1 6 . 3 2} & \mathbf{1 1 . 5 1} & \mathbf{1 4 . 1 2} & \mathbf{1 8 . 6 6}\end{array}$

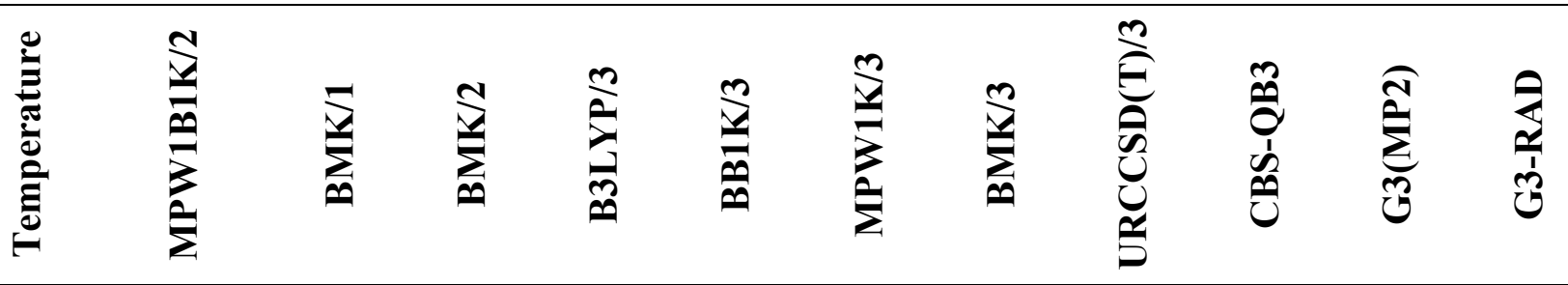

\begin{tabular}{cccccccccccc}
\hline \multicolumn{7}{l}{ Theoretical rate constants $($ In k } \\
600 & 7.75 & 7.00 & 7.26 & 7.73 & 6.60 & 6.92 & 6.60 & 7.04 & 7.78 & 7.63 & 7.55 \\
610 & 7.89 & 7.15 & 7.41 & 7.86 & 6.76 & 7.07 & 6.76 & 7.19 & 7.91 & 7.77 & 7.69 \\
620 & 8.02 & 7.30 & 7.55 & 8.00 & 6.91 & 7.22 & 6.91 & 7.34 & 8.05 & 7.90 & 7.83 \\
630 & 8.15 & 7.44 & 7.68 & 8.12 & 7.05 & 7.36 & 7.05 & 7.47 & 8.18 & 8.03 & 7.96 \\
640 & 8.27 & 7.57 & 7.82 & 8.25 & 7.20 & 7.50 & 7.20 & 7.61 & 8.30 & 8.16 & 8.08 \\
650 & 8.40 & 7.70 & 7.94 & 8.37 & 7.33 & 7.63 & 7.33 & 7.74 & 8.42 & 8.28 & 8.21 \\
660 & 8.51 & 7.83 & 8.07 & 8.49 & 7.47 & 7.76 & 7.47 & 7.87 & 8.54 & 8.40 & 8.33 \\
670 & 8.63 & 7.95 & 8.19 & 8.60 & 7.60 & 7.88 & 7.60 & 7.99 & 8.66 & 8.52 & 8.45 \\
680 & 8.74 & 8.07 & 8.30 & 8.71 & 7.72 & 8.00 & 7.72 & 8.11 & 8.77 & 8.63 & 8.56 \\
690 & 8.85 & 8.19 & 8.42 & 8.82 & 7.84 & 8.12 & 7.84 & 8.23 & 8.87 & 8.74 & 8.67 \\
700 & 8.95 & 8.30 & 8.53 & 8.92 & 7.96 & 8.23 & 7.96 & 8.34 & 8.98 & 8.84 & 8.77 \\
710 & 9.05 & 8.41 & 8.63 & 9.02 & 8.07 & 8.35 & 8.07 & 8.45 & 9.08 & 8.95 & 8.88 \\
720 & 9.15 & 8.52 & 8.74 & 9.12 & 8.19 & 8.45 & 8.19 & 8.55 & 9.18 & 9.05 & 8.98 \\
730 & 9.25 & 8.62 & 8.84 & 9.22 & 8.29 & 8.56 & 8.29 & 8.66 & 9.28 & 9.14 & 9.08 \\
740 & 9.34 & 8.72 & 8.94 & 9.31 & 8.40 & 8.66 & 8.40 & 8.76 & 9.37 & 9.24 & 9.17 \\
750 & 9.43 & 8.82 & 9.03 & 9.40 & 8.50 & 8.76 & 8.50 & 8.86 & 9.46 & 9.33 & 9.26
\end{tabular}


TABLE S7: continued ...

$\begin{array}{llllllllllll}760 & 9.52 & 8.92 & 9.13 & 9.49 & 8.60 & 8.86 & 8.60 & 8.95 & 9.55 & 9.42 & 9.35 \\ 770 & 9.61 & 9.01 & 9.22 & 9.57 & 8.70 & 8.95 & 8.70 & 9.04 & 9.63 & 9.51 & 9.44 \\ 780 & 9.69 & 9.10 & 9.30 & 9.66 & 8.80 & 9.04 & 8.80 & 9.13 & 9.72 & 9.59 & 9.53 \\ 790 & 9.78 & 9.19 & 9.39 & 9.74 & 8.89 & 9.13 & 8.89 & 9.22 & 9.80 & 9.67 & 9.61 \\ 800 & 9.86 & 9.28 & 9.47 & 9.82 & 8.98 & 9.22 & 8.98 & 9.31 & 9.88 & 9.75 & 9.69\end{array}$

$f_{k}$ values comparing $k_{\text {theory }}$ with the $k_{\text {expt }}$ values of Tokmakov et al. [22]

$\begin{array}{lrrrrrrrrrrr}600 & 11.82 & 5.62 & 7.30 & 11.57 & 3.77 & 5.19 & 3.77 & 5.85 & 12.18 & 10.51 & 9.70 \\ 610 & 11.46 & 5.50 & 7.12 & 11.21 & 3.71 & 5.09 & 3.71 & 5.73 & 11.80 & 10.20 & 9.42 \\ 620 & 11.12 & 5.40 & 6.95 & 10.86 & 3.67 & 5.00 & 3.67 & 5.62 & 11.45 & 9.91 & 9.17 \\ 630 & 10.80 & 5.30 & 6.80 & 10.54 & 3.62 & 4.91 & 3.62 & 5.51 & 11.11 & 9.63 & 8.92 \\ 640 & 10.50 & 5.20 & 6.65 & 10.24 & 3.57 & 4.83 & 3.57 & 5.41 & 10.80 & 9.37 & 8.69 \\ 650 & 10.22 & 5.11 & 6.50 & 9.95 & 3.53 & 4.75 & 3.53 & 5.31 & 10.51 & 9.13 & 8.47 \\ 660 & 9.95 & 5.02 & 6.37 & 9.69 & 3.49 & 4.67 & 3.49 & 5.21 & 10.23 & 8.89 & 8.27 \\ 670 & 9.70 & 4.94 & 6.24 & 9.43 & 3.45 & 4.60 & 3.45 & 5.12 & 9.97 & 8.68 & 8.07 \\ 680 & 9.46 & 4.86 & 6.12 & 9.19 & 3.41 & 4.53 & 3.41 & 5.04 & 9.72 & 8.47 & 7.89 \\ 690 & 9.23 & 4.78 & 6.00 & 8.97 & 3.38 & 4.46 & 3.38 & 4.96 & 9.48 & 8.27 & 7.71 \\ 700 & 9.02 & 4.71 & 5.89 & 8.75 & 3.34 & 4.40 & 3.34 & 4.88 & 9.26 & 8.09 & 7.55 \\ 710 & 8.81 & 4.64 & 5.79 & 8.55 & 3.31 & 4.34 & 3.31 & 4.80 & 9.05 & 7.91 & 7.39 \\ 720 & 8.62 & 4.57 & 5.69 & 8.35 & 3.28 & 4.28 & 3.28 & 4.73 & 8.85 & 7.74 & 7.24 \\ 730 & 8.44 & 4.51 & 5.59 & 8.17 & 3.25 & 4.23 & 3.25 & 4.66 & 8.66 & 7.58 & 7.10 \\ 740 & 8.26 & 4.45 & 5.50 & 7.99 & 3.22 & 4.17 & 3.22 & 4.60 & 8.47 & 7.43 & 6.96 \\ 750 & 8.09 & 4.39 & 5.41 & 7.82 & 3.19 & 4.12 & 3.19 & 4.54 & 8.30 & 7.28 & 6.83 \\ 760 & 7.93 & 4.33 & 5.33 & 7.67 & 3.16 & 4.07 & 3.16 & 4.48 & 8.13 & 7.14 & 6.70 \\ 770 & 7.78 & 4.28 & 5.25 & 7.51 & 3.13 & 4.02 & 3.13 & 4.42 & 7.98 & 7.01 & 6.58 \\ 780 & 7.63 & 4.22 & 5.17 & 7.37 & 3.11 & 3.98 & 3.11 & 4.36 & 7.83 & 6.88 & 6.47 \\ 790 & 7.49 & 4.17 & 5.09 & 7.23 & 3.08 & 3.93 & 3.08 & 4.31 & 7.68 & 6.76 & 6.36 \\ 800 & 7.36 & 4.13 & 5.02 & 7.10 & 3.06 & 3.89 & 3.06 & 4.26 & 7.54 & 6.64 & 6.25 \\ <\mathbf{f}> & \mathbf{9 . 2 3} & \mathbf{4 . 7 7} & \mathbf{5 . 9 9} & \mathbf{8 . 9 6} & \mathbf{3 . 3 7} & \mathbf{4 . 4 5} & \mathbf{3 . 3 7} & \mathbf{4 . 9 4} & \mathbf{9 . 4 8} & \mathbf{8 . 2 6} & \mathbf{7 . 7 0}\end{array}$

$\mathbf{f}_{\mathrm{k}}$ values comparing $\mathrm{k}_{\text {theory }}$ with the $\mathrm{k}_{\text {expt }}$ values of Heckmann et al. [28]

$\begin{array}{llllllllllll}600 & 1.58 & 0.75 & 0.97 & 1.54 & 0.50 & 0.69 & 0.50 & 0.78 & 1.62 & 1.40 & 1.29 \\ 610 & 1.61 & 0.77 & 1.00 & 1.57 & 0.52 & 0.71 & 0.52 & 0.80 & 1.66 & 1.43 & 1.32 \\ 620 & 1.64 & 0.80 & 1.03 & 1.60 & 0.54 & 0.74 & 0.54 & 0.83 & 1.69 & 1.46 & 1.35 \\ 630 & 1.67 & 0.82 & 1.05 & 1.63 & 0.56 & 0.76 & 0.56 & 0.85 & 1.72 & 1.49 & 1.38 \\ 640 & 1.70 & 0.84 & 1.08 & 1.66 & 0.58 & 0.78 & 0.58 & 0.88 & 1.75 & 1.52 & 1.41 \\ 650 & 1.73 & 0.87 & 1.10 & 1.69 & 0.60 & 0.81 & 0.60 & 0.90 & 1.78 & 1.55 & 1.44 \\ 660 & 1.76 & 0.89 & 1.13 & 1.72 & 0.62 & 0.83 & 0.62 & 0.92 & 1.81 & 1.58 & 1.46 \\ 670 & 1.79 & 0.91 & 1.15 & 1.74 & 0.64 & 0.85 & 0.64 & 0.95 & 1.84 & 1.60 & 1.49 \\ 680 & 1.82 & 0.94 & 1.18 & 1.77 & 0.66 & 0.87 & 0.66 & 0.97 & 1.87 & 1.63 & 1.52 \\ 690 & 1.85 & 0.96 & 1.20 & 1.80 & 0.68 & 0.89 & 0.68 & 0.99 & 1.90 & 1.66 & 1.55 \\ 700 & 1.88 & 0.98 & 1.23 & 1.82 & 0.70 & 0.92 & 0.70 & 1.02 & 1.93 & 1.68 & 1.57\end{array}$


TABLE S7: continued ...

$\begin{array}{llllllllllll}710 & 1.91 & 1.00 & 1.25 & 1.85 & 0.72 & 0.94 & 0.72 & 1.04 & 1.96 & 1.71 & 1.60 \\ 720 & 1.93 & 1.03 & 1.28 & 1.87 & 0.74 & 0.96 & 0.74 & 1.06 & 1.99 & 1.74 & 1.62 \\ 730 & 1.96 & 1.05 & 1.30 & 1.90 & 0.75 & 0.98 & 0.75 & 1.08 & 2.01 & 1.76 & 1.65 \\ 740 & 1.99 & 1.07 & 1.32 & 1.92 & 0.77 & 1.00 & 0.77 & 1.11 & 2.04 & 1.79 & 1.68 \\ 750 & 2.02 & 1.09 & 1.35 & 1.95 & 0.79 & 1.03 & 0.79 & 1.13 & 2.07 & 1.81 & 1.70 \\ 760 & 2.04 & 1.11 & 1.37 & 1.97 & 0.81 & 1.05 & 0.81 & 1.15 & 2.09 & 1.84 & 1.72 \\ 770 & 2.07 & 1.14 & 1.39 & 2.00 & 0.83 & 1.07 & 0.83 & 1.17 & 2.12 & 1.86 & 1.75 \\ 780 & 2.09 & 1.16 & 1.42 & 2.02 & 0.85 & 1.09 & 0.85 & 1.20 & 2.15 & 1.89 & 1.77 \\ 790 & 2.12 & 1.18 & 1.44 & 2.04 & 0.87 & 1.11 & 0.87 & 1.22 & 2.17 & 1.91 & 1.80 \\ 800 & 2.14 & 1.20 & 1.46 & 2.07 & 0.89 & 1.13 & 0.89 & 1.24 & 2.20 & 1.93 & 1.82 \\ <\mathbf{f}> & \mathbf{1 . 8 7} & \mathbf{0 . 9 8} & \mathbf{1 . 2 2} & \mathbf{1 . 8 2} & \mathbf{0 . 7 0} & \mathbf{0 . 9 2} & \mathbf{0 . 7 0} & \mathbf{1 . 0 1} & \mathbf{1 . 9 2} & \mathbf{1 . 6 8} & \mathbf{1 . 5 7}\end{array}$

$f_{k}$ values comparing $k_{\text {theory }}$ with the $k_{\text {expt }}$ values of Duncan et al. [29]

\begin{tabular}{llllllllllll}
600 & 32.07 & 15.24 & 19.79 & 31.38 & 10.21 & 14.08 & 10.21 & 15.88 & 33.02 & 28.50 & 26.31 \\
610 & 31.62 & 15.19 & 19.64 & 30.91 & 10.25 & 14.05 & 10.25 & 15.81 & 32.55 & 28.13 & 26.00 \\
620 & 31.19 & 15.14 & 19.50 & 30.46 & 10.28 & 14.02 & 10.28 & 15.75 & 32.10 & 27.78 & 25.70 \\
630 & 30.78 & 15.09 & 19.36 & 30.04 & 10.31 & 14.00 & 10.31 & 15.69 & 31.67 & 27.44 & 25.42 \\
640 & 30.39 & 15.05 & 19.23 & 29.63 & 10.34 & 13.97 & 10.34 & 15.64 & 31.25 & 27.11 & 25.15 \\
650 & 30.01 & 15.00 & 19.10 & 29.23 & 10.37 & 13.95 & 10.37 & 15.58 & 30.86 & 26.80 & 24.89 \\
660 & 29.65 & 14.96 & 18.98 & 28.86 & 10.40 & 13.92 & 10.40 & 15.53 & 30.48 & 26.50 & 24.64 \\
670 & 29.31 & 14.92 & 18.86 & 28.50 & 10.43 & 13.90 & 10.43 & 15.48 & 30.12 & 26.21 & 24.40 \\
680 & 28.98 & 14.88 & 18.75 & 28.16 & 10.46 & 13.88 & 10.46 & 15.43 & 29.77 & 25.94 & 24.17 \\
690 & 28.66 & 14.84 & 18.64 & 27.83 & 10.48 & 13.86 & 10.48 & 15.38 & 29.44 & 25.67 & 23.95 \\
700 & 28.35 & 14.81 & 18.53 & 27.51 & 10.51 & 13.84 & 10.51 & 15.34 & 29.12 & 25.42 & 23.73 \\
710 & 28.06 & 14.77 & 18.43 & 27.21 & 10.54 & 13.82 & 10.54 & 15.29 & 28.81 & 25.17 & 23.53 \\
720 & 27.78 & 14.74 & 18.33 & 26.91 & 10.56 & 13.80 & 10.56 & 15.25 & 28.51 & 24.94 & 23.33 \\
730 & 27.51 & 14.70 & 18.23 & 26.63 & 10.59 & 13.78 & 10.59 & 15.21 & 28.22 & 24.71 & 23.14 \\
740 & 27.24 & 14.67 & 18.14 & 26.36 & 10.61 & 13.76 & 10.61 & 15.17 & 27.95 & 24.49 & 22.95 \\
750 & 26.99 & 14.64 & 18.05 & 26.10 & 10.63 & 13.74 & 10.63 & 15.13 & 27.68 & 24.28 & 22.77 \\
760 & 26.75 & 14.61 & 17.96 & 25.85 & 10.65 & 13.72 & 10.65 & 15.09 & 27.43 & 24.08 & 22.60 \\
770 & 26.51 & 14.58 & 17.88 & 25.60 & 10.68 & 13.71 & 10.68 & 15.05 & 27.18 & 23.88 & 22.43 \\
780 & 26.29 & 14.55 & 17.79 & 25.37 & 10.70 & 13.69 & 10.70 & 15.02 & 26.94 & 23.69 & 22.27 \\
790 & 26.07 & 14.52 & 17.71 & 25.14 & 10.72 & 13.67 & 10.72 & 14.98 & 26.71 & 23.51 & 22.12 \\
800 & 25.85 & 14.49 & 17.64 & 24.92 & 10.74 & 13.66 & 10.74 & 14.95 & 26.49 & 23.33 & 21.97 \\
$<\mathbf{f}>$ & $\mathbf{2 8 . 4 0}$ & $\mathbf{1 4 . 8 1}$ & $\mathbf{1 8 . 5 4}$ & $\mathbf{2 7 . 5 6}$ & $\mathbf{1 0 . 5 1}$ & $\mathbf{1 3 . 8 4}$ & $\mathbf{1 0 . 5 1}$ & $\mathbf{1 5 . 3 4}$ & $\mathbf{2 9 . 1 6}$ & $\mathbf{2 5 . 4 5}$ & $\mathbf{2 3 . 7 6}$ \\
\hline
\end{tabular}

